\title{
A Control Lyapunov Function Approach to Multiagent Coordination
}

\author{
Petter Ögren, Magnus Egerstedt, Member, IEEE, and Xiaoming Hu, Member, IEEE
}

\begin{abstract}
In this paper, the multiagent coordination problem is studied. This problem is addressed for a class of robots for which control Lyapunov functions can be found. The main result is a suite of theorems about formation maintenance, task completion time, and formation velocity. It is also shown how to moderate the requirement that, for each individual robot, there exists a control Lyapunov function. An example is provided that illustrates the soundness of the method.
\end{abstract}

Index Terms-Coordinated control, Lyapunov methods, mobile robots, multirobot system, robot formation control.

\section{INTRODUCTION}

I $\mathrm{N}$ THIS PAPER, we investigate the problem of how to coordinate a collection of robots in such a way that they maintain a given formation relative to each other. The main assumption about the dynamics of the individual robots that we initially make in this paper is that they have control Lyapunov functions (CLFs). Based on this assumption, an abstract and theoretically sound coordination strategy can be developed.

Multiagent formation control problems have been extensively studied in the literature, and our main contribution is that we use CLFs to define the formation. By doing this, we convert the formation control problem, typically a constrained motion control problem of multiple systems, into a stabilization problem for one single system. By this approach, we neither cast the problem without real dynamics [2], nor with an explicit nonlinear robot model [5]. Instead, we believe that by requiring the existence of CLFs, we can capture at least some aspects of the platform dynamics, while not having to spend our main effort on nonlinear robot control. Thus, we can focus on the coordination problem at a higher level.

In addition to the CLF approach, we use the idea of virtual vehicles discussed in [6]. Concepts of similar flavor are the "action reference" suggested by Kang et al. [7] and the "dynamic coordination variable" proposed by Beard et al. [3]. Furthermore, in the terminology of Beard et al. in [9], our approach would fall

Manuscript received June 11, 2001; revised February 7, 2002. This paper was recommended for publication by Associate Editor E. Pagello and Editor S. Hutchinson upon evaluation of the reviewers' comments. This work was supported in part by the SSF through the Centre for Autonomous Systems at the Royal Institute of Technology (KTH), Stockholm, Sweden. This paper was presented in part at IEEE CDC'01, Orlando, FL, December, 2001

P. Ögren and X. Hu are with the Division of Optimization and Systems Theory, Royal Institute of Technology (KTH) SE-100 44 Stockholm, Sweden (e-mail: petter@math.kth.se; hu@math.kth.se).

M. Egerstedt is with the School of Electrical and Computer Engineering, Georgia Institute of Technology, Atlanta, GA 30332 USA (e-mail magnus@ece.gatech.edu).

Digital Object Identifier 10.1109/TRA.2002.804500 into the category of "virtual structures." The formation function we introduce has similarities with the task function of [13], but there is no connection with Lyapunov theory in that approach.

The motivation for studying this type of multiagent coordination problem mainly stems from the observation that there is robustness and strength in numbers. If more than one agent is asked to carry out a given task, e.g., search a disaster area, the likelihood of success increases as more agents are included in the mission. In other situations, cost and energy efficiency indicates that using many small robots might be more beneficial than using one big robot.

The outline of this paper is as follows. In Section II, we define what we mean by a formation, and show how this can be formalized in terms of a formation function. We define a subclass called Lyapunov formation functions, and show how these can be constructed. Next, in Section III, we add error feedback to the time evolution of the formation. In Section IV, we then prove a suite of theorems about bounded formation errors, task completion times, and group velocities. We conclude, in Section $\mathrm{V}$, with an example, illustrating the usefulness of our proposed method.

\section{FORMATION FUNCTIONS}

Our primary object of study is a collection of $m$ robots, whose dynamics can be described by the following set of controlled differential equations:

$$
\dot{x}_{i}=f_{i}\left(x_{i}\right)+g_{i}\left(x_{i}\right) u_{i}, \quad i=1, \ldots, m
$$

where $f_{i}, g_{i} \in C^{\infty}, x_{i} \in \mathbb{R}^{n}$, and $u_{i} \in \mathbb{R}^{p_{i}}$.

Now, a desired formation in $\mathbb{R}^{n m}$ is simply a set $\left\{x_{10}, \ldots x_{m 0}\right\} \in \mathbb{R}^{n m}$, and we define this set implicitly through the null set of a so-called formation function.

Definition II.1-Formation Function: Given a formation $\left\{x_{10}(s), \ldots, x_{m 0}(s)\right\} \in \mathbb{R}^{n m}$ parameterized by a scalar $s$. We say that a positive definite, continuously differentiable map $F: \mathbb{R} \times \mathbb{R}^{n m} \rightarrow \mathbb{R}$ is a formation function to the set $\left\{x_{10}(s), \ldots, x_{m 0}(s)\right\}$ if $\left\{s, x_{1}, \ldots, x_{m}\right\} \in F^{-1}(0)$ gives a unique $\left\{x_{1}, \ldots, x_{m}\right\}$ for each choice of $s \in \mathbb{R}$.

Example II.1: One choice is $F\left(s, x_{1}, \ldots, x_{m}\right)=$ $\Sigma_{i}\left\|x_{i}-x_{i 0}(s)\right\|^{2}$. Clearly, $F=0$ implies a unique $x_{i}$ for each $s$, as demanded in the definition. But as we will see, robot dynamics (Definition II.2) can be taken into account when choosing $F(s, x)$.

In order to focus on the high-level coordination issues, we connect the formation function with the concept of CLFs. 
Definition II.2-Lyapunov Formation Function: A formation function $F(s, x)$ is a Lyapunov formation function if there exists a class $\mathcal{K}$ function $\sigma$ and a control $u(s, x)$, such that

$$
\frac{-\left(\frac{\partial F}{\partial x}\right)^{T} \dot{x}}{\sigma(F(s, x))} \geq 1 \quad \forall s \in \mathbb{R}, \quad x \in \mathbb{R}^{n m} \mid 0<F(s, x) \leq F_{U} .
$$

Furthermore, the left-hand side of this expression goes to infinity as $F$ approaches zero.

A class $\mathcal{K}$ function is a function $\sigma:[0, k) \rightarrow \mathbb{R}_{+}$, continuous, strictly increasing, and satisfying $\sigma(0)=0, \sigma(x)>0, \forall x>0$ [11].

To set the stage for our main existence theorem on Lyapunov formation functions, we now state two lemmas.

Lemma II.1-Locally Positive Definite (LPD) Implications: If the Lyapunov function $V$ is LPD and decresent and $-\dot{V}$ is LPD, then the bound of Definition II.2 is fulfilled.

Proof: The conditions on $V$ implies [11] that there exist class $\mathcal{K}$ functions $\alpha, \beta$, and $\gamma$, such that

$$
\begin{aligned}
& \alpha(\|x\|) \leq V(x) \leq \beta(\|x\|) \\
& \gamma(\|x\|) \leq-\dot{V} .
\end{aligned}
$$

Since the functions are of class $\mathcal{K}$, they are all invertible and positive, and we have $\|x\| \geq \beta^{-1}(V(x))$ and

$$
-\dot{V} \geq \gamma(\|x\|) \geq \gamma\left(\beta^{-1}(V(x))\right)=\sigma(V(x)) .
$$

Note that this makes $\sigma$ a class $\mathcal{K}$ function. Now we have

$$
\frac{-\dot{V}}{\sigma(V)} \geq 1
$$

and we can choose $\sigma(V)=\gamma\left(\beta^{-1}(V(x))\right)$. Furthermore, if the property only holds locally, i.e., when $\|x\| \leq \epsilon$, then this condition can be replaced by $V(x) \leq \alpha(\epsilon)=V_{U}$, since $\|x\| \leq$ $\alpha^{-1}(V(x))$.

Remark II.1: These are the standard Lyapunov assumptions for showing that a system is asymptotically stable [11]. The case of a semidefinite Lyapunov function for the whole formation is discussed in [12].

Lemma II.2-Limit Property: Given a $\sigma$ such that

$$
\frac{-\left(\frac{\partial F}{\partial x}\right)^{T} \dot{x}}{\sigma(F(s, x))} \geq 1 \quad \forall s \in \mathbb{R}, \quad x \in \mathbb{R}^{n m} \mid 0<F(s, x) \leq F_{U} .
$$

The limit property of Definition II.2 will be fulfilled if we choose a new $\sigma^{\prime}=\left(\sigma^{2}\right) /(1+\sigma)$.

Proof: Since $\sigma^{\prime} \leq \sigma$ the bound still holds. Furthermore

$$
\begin{aligned}
\frac{-\left(\frac{\partial F}{\partial x}\right)^{T} \dot{x}}{\sigma^{\prime}(F(s, x))} & =\frac{-\left(\frac{\partial F}{\partial x}\right)^{T} \dot{x}}{\sigma(F(s, x))} \frac{1+\sigma(F(s, x))}{\sigma(F(s, x))} \\
& \geq \frac{1+\sigma(F(s, x))}{\sigma(F(s, x))}
\end{aligned}
$$

which clearly approaches infinity as $F \rightarrow 0$.

Remark II.2: The local stability property of Definition II.2 in combination with the feedback of (3) in Section III will be shown to guarantee successful traversal of the whole trajectory.

Remark II.3: In general, finding a CLF is an open problem, however, it is known that CLFs exist for a large class of prac- tically important systems [8], including feedback linearizable systems (as will be seen in Section V). Note also the Artstein Sonntag theorem on existence of CLFs [1].

We now go on to state and prove the main existence theorem of Lyapunov formation functions.

Theorem II.1-Lyapunov Formation Function: If the $n$ vehicles have translationally invariant (in position coordinates) dynamics and LPD CLFs $V_{i}$, with locally negative definite time derivatives, then we can form a Lyapunov formation function by a weighted sum of the parameterized CLFs

$$
F(s, x)=\sum_{i=1}^{m} \beta_{i} V_{i}\left(s, x_{i}\right) .
$$

Proof: By Lemma II.1, the bound of Definition II. 2 holds, pointwise. The hypothesis that the vehicle dynamics are invariant with respect to position translations lets us parameterize them with respect to $s, V_{i}(s, x)$, and we have that

$$
\frac{-\left(\frac{\partial V_{i}}{\partial x_{i}}\right)^{T} \dot{x}_{i}}{\sigma_{i}\left(V_{i}\left(s, x_{i}\right)\right)} \geq 1 \quad \forall s \in \mathbb{R}, \quad x_{i} \in \mathbb{R}^{n} \mid V_{i}\left(s, x_{i}\right) \leq V_{U i}
$$

where the $V_{U i}$ are given by $\alpha(\epsilon)$ as in Lemma II.1.

We have that

$$
\begin{aligned}
-\left(\frac{\partial F}{\partial x}\right)^{T} \dot{x} & =\sum_{i=1}^{m} \beta_{i}\left(-\frac{\partial V_{i}}{\partial x}\right)^{T} \dot{x} \\
& \geq \sum_{i=1}^{m} \beta_{i} \sigma_{i}\left(V_{i}(s, x)\right) \\
& \geq \min _{i} \beta_{i} \sigma_{i}\left(\frac{F}{\beta_{\max } m}\right) \\
& =\sigma(F(s, x))
\end{aligned}
$$

where

$$
\sigma(F(s, x))=\min _{i} \beta_{i} \sigma_{i}\left(\frac{F}{\beta_{\max } m}\right) .
$$

The second inequality above follows, since there exists a $j$ such that $V_{j} \geq\left(F / \beta_{\max } m\right)$, and thus

$$
\sigma_{j}\left(V_{j}(s, x)\right) \geq \min _{i} \sigma_{i}\left(\frac{F}{\beta_{\max } m}\right) .
$$

The limit property follows from Lemma II.2.

Remark II.4: Note that the different vehicles in the formation can have completely different dynamics [and therefore Lyapunov functions $V_{i}\left(s, x_{i}\right)$ in (2)]. Furthermore, the choice of coefficients in (2) reflects how large deviations from zero are allowed for each $V_{i}$.

\section{COORDINATED CONTROL}

By establishing these observations about the Lyapunov formation functions derived from the individual CLFs, we can now shift our attention to actually controlling the evolution of the formation. The one parameter that we can control is the $s$ parameter, i.e., the parameterization of the time evolution of the desired positions. We do this by specifying the trajectory that we want the so-called virtual leader, $x_{0}(s(t))$, to follow. 
This nonphysical leader is a reference point in the state space with respect to which we can define the rest of the formation. We denote the trajectory executed by the virtual leader by $x_{0}(s(t))=p(s(t))$. Intuitively, one might want to set $s(t)=t$. But, due to robustness considerations, we incorporate error feedback into the time evolution of $s$ (see, for example, [6]) and let $\dot{s}$ be given by

$$
\dot{s}=\min \left\{\frac{v_{0}}{\delta+\left\|\frac{\partial p(s)}{\partial s}\right\|}, \frac{-\left(\frac{\partial F}{\partial x}\right)^{T} \dot{x}}{\delta+\left|\frac{\partial F}{\partial s}\right|}\left(\frac{\sigma\left(F_{U}\right)}{\sigma(F(s, x))}\right)\right\} .
$$

Here, $\delta>0$ is a small positive constant that prevents $\dot{s}$ from becoming singular, and $F_{U}$ is the bound of Definition II.2 or something smaller chosen by the user. It will be shown to be an upper bound on the Lyapunov formation function $F(s, x)$. The idea is to say that the formation is being respected as long as $F(s, x) \leq F_{U} . \sigma$ is the class $\mathcal{K}$ function of Definition II.2. Furthermore, $v_{0}$ is the nominal velocity that we want the formation to move with, and as we will see later, it holds that $\left\|\dot{x}_{0}(s(t))\right\| \approx v_{0}$ when $F$ is small.

\section{Theoretical Properties}

In the following paragraphs we will investigate what theoretical properties the evolution of the multi-agent formation exhibits when letting $\dot{s}$ be given by (3). We will show that if $F\left(s\left(t_{0}\right), x\left(t_{0}\right)\right) \leq F_{U}$, then $F$ remains bounded by $F_{U}$ along trajectories for all times greater than $t_{0}$. We will also show that if $s \in\left[s_{\text {start }}, s_{\text {final }}\right]$ and if $\dot{s}$ is governed by (3), then $s$ reaches $s_{\text {final }}$ in finite time. We will conclude our theoretical investigations by showing that $\left\|\dot{x}_{0}(s(t))\right\| \approx v_{0}$ if the formation function is small enough.

Theorem IV.1-Error Bound: If $F\left(s\left(t_{0}\right), x\left(t_{0}\right)\right) \leq F_{U}$, then

$$
F(s, x) \leq F_{U}, \quad \forall t \geq t_{0}
$$

i.e., the Lyapunov formation function will never exceed $F_{U}$.

Proof: We directly have

$$
\begin{aligned}
\dot{F} & =\left(\frac{\partial F}{\partial x}\right)^{T} \dot{x}+\frac{\partial F}{\partial s} \dot{s}(s, x) \\
& \leq\left(\frac{\partial F}{\partial x}\right)^{T} \dot{x}+\frac{\partial F}{\partial s} \frac{-\left(\frac{\partial F}{\partial x}\right)^{T} \dot{x}}{\delta+\left|\frac{\partial F}{\partial s}\right|}\left(\frac{\sigma\left(F_{U}\right)}{\sigma(F(s, x))}\right) \\
& =\left(\frac{\partial F}{\partial x}\right)^{T} \dot{x}\left(1-\frac{\frac{\partial F}{\partial s}}{\delta+\left|\frac{\partial F}{\partial s}\right|}\left(\frac{\sigma\left(F_{U}\right)}{\sigma(F(s, x))}\right)\right) .
\end{aligned}
$$

To show that the set $\left\{(s, x) \mid F(s(t), x(t)) \leq F_{U}\right\}$ is invariant, we will note that for any $\left(s\left(t_{0}\right), x\left(t_{0}\right)\right)$ such that $F\left(s\left(t_{0}\right), x\left(t_{0}\right)\right) \geq F_{U}$, we have

$$
\dot{F}\left(s\left(t_{0}\right), \quad x\left(t_{0}\right)\right) \leq 0 .
$$

For such a $\left(s\left(t_{0}\right), x\left(t_{0}\right)\right)$, it holds that

$$
\begin{aligned}
& \frac{\sigma\left(F_{U}\right)}{\sigma\left(F\left(s\left(t_{0}\right), x\left(t_{0}\right)\right)\right)} \leq 1 \\
& \frac{\frac{\partial F\left(s\left(t_{0}\right), x\left(t_{0}\right)\right)}{\partial s}}{\left(\delta+\left|\frac{\partial F\left(s\left(t_{0}\right), x\left(t_{0}\right)\right)}{\partial s}\right|\right)} \leq 1
\end{aligned}
$$

and

$$
\left(\frac{\partial F\left(s\left(t_{0}\right), x\left(t_{0}\right)\right)}{\partial x}\right)^{T} \dot{x}\left(t_{0}\right) \leq 0
$$

This directly gives that if $F\left(s\left(t_{0}\right), x\left(t_{0}\right)\right) \geq F_{U}$, then $\dot{F}(s, x) \leq$ 0 along trajectories. Thus, the set $\left\{(s, x) \mid F(s(t), x(t)) \leq F_{U}\right\}$ is invariant, i.e., if $F\left(s\left(t_{0}\right), x\left(t_{0}\right)\right) \leq F_{U}$, then $F(s(t), x(t)) \leq$ $F_{U}$ for all $t \geq t_{0}$.

Remark IV.1: In most cases, a careful choice of $F_{U}$ can guarantee collision avoidance.

Theorem IV.2-Finite Completion Time: If the trajectory is given as an interval, $s \in\left[s_{\text {start }}, s_{\text {final }}\right]$, then by using the controller in (3) we can find an upper bound $M<\infty$, such that

$$
T_{\text {final }} \leq M .
$$

In other words, there is an upper bound on the completion time.

Proof: Let the completion time be defined in such a way that

$$
\int_{T_{\text {start }}}^{T_{\text {final }}} \dot{s}=s_{\text {final }}-s_{\text {start }} .
$$

If there exists a constant $v_{s}>0$ such that $\dot{s} \geq v_{s}$, then we obviously have

$$
T_{\text {final }} \leq \frac{s_{\text {final }}-s_{\text {start }}}{v_{s}}+T_{\text {start }} .
$$

The proof thus consists of finding such a lower bound on $\dot{s}$. Let

$$
\begin{aligned}
& K_{1}=\max _{s \in\left[s_{\text {start }}, s_{\text {final }}\right]}\left\{\delta+\left\|\frac{\partial p(s)}{\partial s}\right\|\right\} \\
& K_{2}=\max _{s \in\left[s_{s}, s_{f}\right], x \in \mathbb{R}^{n m} \mid F(s, x) \leq F_{U}}\left\{\delta+\left|\frac{\partial F(s, x)}{\partial s}\right|\right\} .
\end{aligned}
$$

We have that

$$
\begin{aligned}
\dot{s}(s, x) & =\min \left\{\frac{v_{0}}{\delta+\left\|\frac{\partial p(s)}{\partial s}\right\|}, \frac{-\left(\frac{\partial F}{\partial x}\right)^{T} \dot{x}}{\delta+\left|\frac{\partial F}{\partial s}\right|}\left(\frac{\sigma\left(F_{U}\right)}{\sigma(F(s, x))}\right)\right\} \\
& \geq \min \left\{\frac{v_{0}}{K_{1}}, \frac{L \sigma\left(F_{U}\right)}{K_{2}}\right\}=v_{s}>0
\end{aligned}
$$

since $\left(F_{U}\right) /(F(s, x)) \geq 1$. Thus

$$
T_{\text {final }} \leq \frac{s_{\text {final }}-s_{\text {start }}}{v_{s}}+T_{\text {start }}=M
$$

which concludes the proof.

Theorem IV.3-Formation Velocity: If the formation error is small, $F(s, x) \ll F_{U}$ and $\delta v_{0} \ll\|\partial p(s) / \partial s\|$, then

$$
\left\|\frac{d x_{0}}{d t}\right\| \approx v_{0}
$$

i.e., the formation velocity is $v_{0}$.

Proof: We will start by showing that the right-hand term in the brackets of (3) grows to infinity as $F$ approaches zero

$$
\begin{array}{r}
\frac{-\left(\frac{\partial F}{\partial x}\right)^{T} \dot{x}}{\delta+\left|\frac{\partial F}{\partial s}\right|}\left(\frac{\sigma\left(F_{U}\right)}{\sigma(F(s, x))}\right) \geq \frac{\sigma\left(F_{U}\right)}{K_{2}} \frac{-\left(\frac{\partial F}{\partial x}\right)^{T} \dot{x}}{\sigma(F(s, x))} \rightarrow \infty \\
\text { as } F(s, x) \rightarrow 0
\end{array}
$$


by Assumption II.2. Thus, the left-hand term governs $\dot{s}$ when $F$ is small. In that case, we have

$$
\begin{aligned}
\left\|\frac{d x_{0}}{d t}\right\| & =\left\|\frac{\partial p(s)}{\partial s}\right\| \dot{s} \\
& =\left\|\frac{\partial p(s)}{\partial s}\right\| \frac{v_{0}}{\delta+\left\|\frac{\partial p(s)}{\partial s}\right\|} \\
& =v_{0}-\frac{\delta v_{0}}{\delta+\left\|\frac{\partial p(s)}{\partial s}\right\|} \approx v_{0} .
\end{aligned}
$$

\section{SimUlation EXAMPLES}

We will now go on to illustrate Theorem IV.I and Theorem $I V .3$, as well as the effect of measurement noise on our proposed approach. In the following example, we model the robots using the standard unicycle model (see, for example, [4] and [5]). Such a model is applicable to the Nomadic Scout, which is the robot we work with at the Royal Institute of Technology in Stockholm. It is, furthermore, adequate for most all-terrain caterpillar vehicles as well.

The equations of motion are

$$
\dot{z}_{1}=v \cos \theta, \quad \dot{z}_{2}=v \sin \theta, \quad \dot{\theta}=\omega, \quad m \dot{v}=F, \quad J \dot{\omega}=\tau
$$

where $\left(z_{1}, z_{2}\right)$ is the center of the wheel axis, $\theta$ is the directional angle, and $v, \omega$ are forward and angular velocities. The controls $F, \tau$ are the applied force and torque. We choose the output to be the position of an off-axis point, $x_{1}=z_{1}+l \cos (\theta), x_{2}=$ $z_{2}+l \sin (\theta)$, perhaps the center of gravity.

It was shown in [9] that this model can be feedback linearized to a two-dimensional double integrator $\ddot{x}_{1}=u_{1}, \ddot{x}_{2}=u_{2}$. (This property was also used implicitly in [5]). A parameterized CLF and feedback control of a one-dimensional double integrator is

$$
\begin{aligned}
V_{i}\left(s, x_{i}\right) & =\left(x_{i}-p_{i}(s)\right)^{2}+\left(\left(x_{i}-p(s)\right)+\dot{x}_{i}\right)^{2} \\
u_{i} & =0.5\left(-\left(x_{i}-p_{i}(s)\right)-3 \dot{x}_{i}\right)
\end{aligned}
$$

yielding $\dot{V}_{i}=-\left(x_{i}-p_{i}(s)\right)^{2}-\dot{x}_{i}^{2}$. We note that there is some flexibility in choosing the second term in $V_{i}$, since the only hard formation constraint is $x_{i}=p_{i}(s)$.

Now we can choose $F(s, x)=\sum_{i=1}^{m} V_{i}\left(s, x_{i}\right)$, as in (2), and it is straightforward to check that $\sigma(z)=\left((0.3 z)^{2}\right) /(0.3 z+1)$ fulfills the bound and limit property of Definition II.2.

A formation of three feedback linearized dynamic unicycles thus gives a 12-dimensional system (Fig. 1). In the first part of the simulation, the three desired trajectories meet to form a side-by-side formation. When the robots are close to horizontal coordinate $6 \mathrm{~m}$, we increase $v_{0}=0.1$ to $v_{0}=0.3$. This will drive the formation function close to, but not above, the upper limit of $F_{U}=1$. When this happens, the velocity is decreased to a value below $v_{0}=0.3$. Finally, in the last part, when passing the 12-m mark, we introduce a stochastic measurement error in the control of the topmost robot. Since we are already close to the upper bound $F_{U}=1$, the disturbance makes the whole formation slow down when needed (as seen in the lowest plot) to respect the bound. This is not guaranteed by Theorem IV.1, since the proof is only valid in a deterministic setting. However,

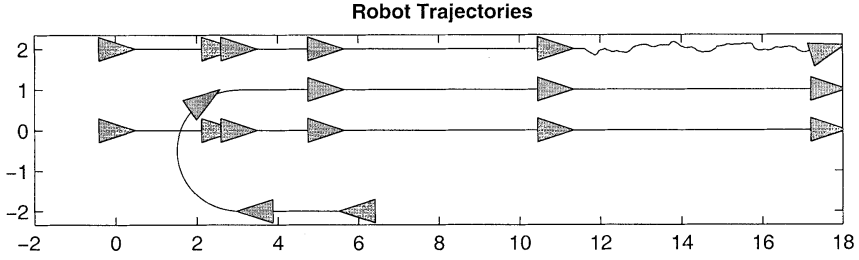

(a)

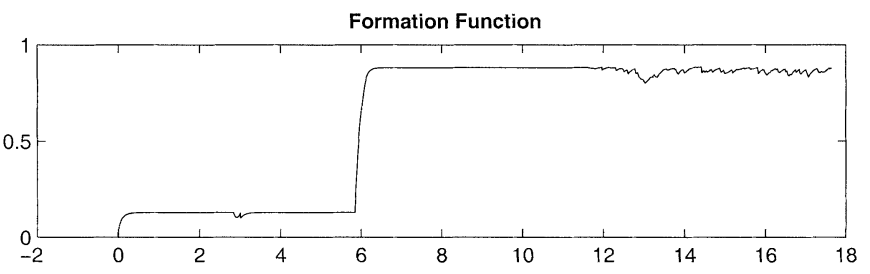

(b)

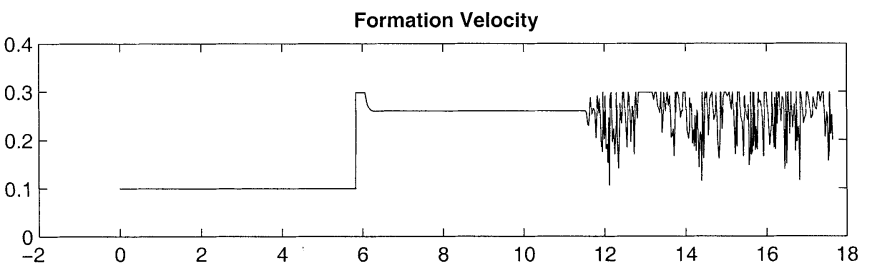

(c)

Fig. 1. (a) Top view of the robot trajectories. (b) The formation function. (c) The formation velocity. The horizontal axis of the two lower plots refer to the horizontal position of the topmost robot.

the successful simulation does indicate some robustness of the approach.

\section{CONCLUSION}

In this paper, we propose a stable coordination strategy for a team of formation constrained autonomous agents. A Lyapunov formation function defined under standard assumptions can be constructed from individual-CLFs. The Lyapunov formation function is used to prove properties such as formation maintenance, task completion time, and formation velocity. Finally, we present an example that illustrates the soundness of our method.

\section{ACKNOWLEDGMENT}

The authors would like to thank the three anonymous reviewers for their insightful comments.

\section{REFERENCES}

[1] Z. Arstein, "Stabilization with relaxed controls," Nonlinear Anal., vol. 7, no. 11, pp. 1163-1173, 1983.

[2] T. Balch and R. C. Arkin, "Behavior-based formation control for multirobot teams," IEEE Trans. Robot. Automat., vol. 14, pp. 926-939, Dec. 1998.

[3] R. W. Beard, J. Lawton, and F. Y. Hadaegh, "A coordination architecture for spacecraft formation control," IEEE Trans. Control Syst. Technol., to be published.

[4] C. Canudas de Wit, B. Siciliano, and G. Bastin, Theory of Robot Control. Berlin, Germany: Springer-Verlag, 1996.

[5] J. Desai, J. Ostrowski, and V. Kumar, "Control of formations for multiple robots," Proc. IEEE Int. Conf. Robotics and Automation, vol. 4, pp. 2864-2869, May 1998.

[6] M. Egerstedt, X. Hu, and A. Stotsky, "Control of a car-like robot using a virtual vehicle approach," presented at the 37th IEEE Conf. Decision and Control, Tampa, FL, Dec. 1998. 
[7] W. Kang, N. Xi, and A. Sparks, "Formation control of autonomous agents in 3D workspace," presented at the IEEE Int. Conf. Robotics and Automation, San Francisco, CA, Apr. 2000.

[8] M. Krstic, I. Kanellakopoulos, and P. Kokotovic, Nonlinear and Adaptive Control Design. New York: Wiley, 1995.

[9] J. R. T. Lawton, B. J. Young, and R. W. Beard, "A decentralized approach to elementary formation maneuvers," IEEE Trans. Robot. Automat., to be published.

[10] P. Ögren, M. Egerstedt, and X. Hu, "A control Lyapunov function approach to multiagent coordination," presented at the IEEE Conf. Decision and Control, Orlando, FL, Dec. 2001.

[11] H. K. Khalil, Nonlinear Systems. Englewood Cliffs, NJ: Prentice-Hall, 1996.

[12] P. Ögren, E. Fiorelli, and N. Leonard, "Formations with a mission: Stable coordination of vehicle group maneuvers," presented at the 15th Int Symp. Mathematical Theory of Networks and Systems, South Bend, IN Aug. 2002.

[13] C. Samson, M. Le Borgne, and B. Espiau, Robot Control, the Task Function Approach, ser. Oxford Engineering Science Series. Oxford, U.K.: Clarendon, 1991.

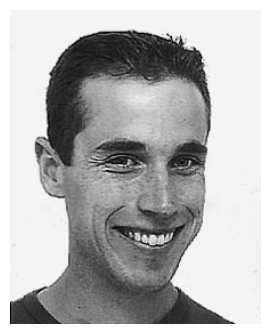

Petter Ögren was born in Stockholm, Sweden, in 1974. He received the M.S. degree in engineering physics in 1998 from the Royal Institute of Technology (KTH), Stockholm, Sweden, where he is currently working toward the Ph.D. degree in the Division of Optimization and Systems Theory. His research interests include multirobot systems, navigation, and obstacle avoidance.

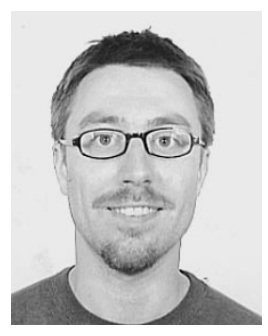

Magnus Egerstedt (S'99-M'00) was born in Stockholm, Sweden, in 1971. He received the B.A. degree in philosophy from Stockholm University, Stockholm, Sweden, in 1996, and the M.S. degree in engineering physics and Ph.D. degree in applied mathematics from the Royal Institute of Technology (KTH), Stockholm, Sweden, in 1996 and 2000, respectively.

He is currently an Assistant Professor in Electrical and Computer Engineering with the Georgia Institute of Technology, Atlanta. In 2000-2001, he was a Postdoctoral Fellow at the Division of Engineering and Applied Science, Harvard University, Cambridge, MA. His research interests include optimal control as well as modeling and analysis of hybrid and discrete event systems, with emphasis on motion planning and control of mobile robots.

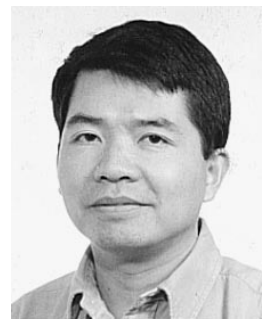

Xiaoming Hu (M'90) was born in Chengdu, China in 1961. He received the B.S. degree from the University of Science and Technology, Hefei, China, in 1983, and the M.S. and Ph.D. degrees from Arizona State University, Tempe, in 1986 and 1989, respectively.

He was a Research Assistant with the Institute of Automation, Academia Sinica, Beijing, China, from 1983 to 1984. From 1989 to 1990, he was a Gustafsson Postdoctoral Fellow at the Royal Institute of Technology, Stockholm, Sweden, where he is currently an Associate Professor. His current research interests are in nonlinear feedback stabilization, nonlinear observer design, sensing and active perseption, motion planning and control of mobile robots, and mobile manipulation. 